\title{
Current Recommendations on Atrial Fibrillation: A Comparison of the Recent European and Canadian Guidelines
}

\author{
Sercan Okutucu ${ }^{\mathrm{a}}$ Bulent Gorenek ${ }^{\mathrm{b}}$ \\ aDepartment of Cardiology, Memorial Ankara Hospital, Memorial Healthcare Group, Ankara, Turkey; ${ }^{\text {b Department of }}$ \\ Cardiology, Faculty of Medicine, Eskisehir Osmangazi University, Eskisehir, Turkey
}

\section{Keywords}

Anticoagulation - Atrial fibrillation - Canadian guidelines .

European guidelines $\cdot$ Rate control $\cdot$ Rhythm control

\begin{abstract}
Background: Guidelines for the diagnosis and management of atrial fibrillation (AF) are frequently published and updated, reflecting the rapid evolution in AF pathogenesis and treatment modalities. Summary: Recently, 2 important guidelines for the diagnosis and management of atrial AF have been published by the European Society of Cardiology (ESC), and Canadian Cardiovascular Society (CCS). Although the evidence-based recommendations and statements are quite similar, there are some important differences between the ESC and CCS guidelines for AF. Herein, we compared the current recommendations and highlighted the differences from the most recent guidelines for AF. Key Messages: Specifically, key differences can be observed in methods evaluating the recommendations; classifications and the definitions; the symptom score used to guide management decisions, longitudinal patient assessment, and structured characterization; the stroke risk stratification algorithm used to determine indications for oral anticoagulation therapy;
\end{abstract}

the role of acetylsalicylic acid in stroke prevention in AF; the antithrombotic regimens that are employed in the setting of chronic coronary syndromes, acute coronary syndromes, and percutaneous coronary intervention; the target heart rate for rate control; and the algorithms for integrated or holistic management of AF. Differences are observed, particularly when the quality of evidence is moderate or low. More research and randomized controlled studies on major gaps identified in current guidelines will further clarify and modify our future management strategies in AF.

(c) 2021 S. Karger AG, Basel

\section{Introduction}

Atrial fibrillation (AF), the most common sustained cardiac arrhythmia, is associated with reduced quality of life (QoL), functional status, cardiac performance, and overall survival $[1,2]$. AF poses significant burden to patients, physicians, and healthcare systems globally. Substantial research has been directed toward getting comprehensive information about the mechanisms behind $\mathrm{AF}$, its natural course and effective therapies, and new evidence is constantly obtained and published $[1,2]$. The karger@karger.com

www.karger.com/crd

Karger ${ }^{\prime \prime}=$$$
\stackrel{2}{2}
$$

(C) 2021 S. Karger AG, Basel
Correspondence to:

Sercan Okutucu, sercanokutucu@yahoo.com 
complexity of AF necessitates a multidimensional and multidisciplinary strategy for the management of AF. The contemporary management of AF is centered on symptomatic improvement, reduction in AF-related hospitalizations, and reduction in stroke or systemic thromboembolism morbidity, heart failure, and mortality. To assist clinicians in the management of AF a multitude of evidence-based guidelines has been published. Recently, 2 important guidelines have been published on AF, namely, 2020 European Society of Cardiology (ESC) guidelines for the diagnosis and management of AF developed in collaboration with the European Association for CardioThoracic Surgery [1] and the 2020 Canadian Cardiovascular Society (CCS)/Canadian Heart Rhythm Society comprehensive guidelines for the management of AF [2]. Although many of the recommendations of these 2 documents are quite similar, there are some differences in the methodologies underlying their development and the specific content. Herein, we compared the current main recommendations from the ESC and CCS guidelines for AF.

\section{Strength of Recommendations and Quality of Evidence}

Both guidelines differ from each other with the methods evaluating the strength of recommendations and quality of evidence. The ESC guidelines for AF categorize recommendations by the conventional class of recommendation and level of evidence. The class of recommendation reflects the magnitude of benefit over risk and corresponds to the strength of the recommendation. The level of evidence denotes the confidence in or certainty of the evidence supporting the recommendation, based on the type, size, quality, and consistency of pertinent research findings $[1,3]$. The CCS guidelines use the Grading of Recommendation Assessment Development and Evaluation (GRADE) process, which specifies the strength of recommendations, and the quality of evidence. The strength of recommendation was graded as "strong" (the desirable effects outweigh the undesirable effects, and therefore, most individuals will be best served by the recommended course of action) or "weak" (the desirable effects probably outweigh the undesirable effects, indicating that there is a need to consider the individual patient's clinical details, circumstances, values, and preferences). These estimates were further refined through detailed appraisal into 4 categorical grades ("high," "moderate," "low," and "very low") after consideration of the risk of bias, confounding, consistency of results, directness of evidence, precision, publication bias, magnitude of effect, and dose-response gradient $[2,4]$.

\section{Classifications and Definitions}

Both of the ESC and CCS guidelines classify AF based on duration and persistence, with or without efforts to restore sinus rhythm. Each describes several patterns of AF. The ESC and CCS guidelines recommend that the 1st presentation of AF be considered "1st diagnosed" (ESC) or "newly diagnosed" (CCS) irrespective of the duration of the arrhythmia or the presence/severity of AF-related symptoms $[1,2]$. Thereafter, AF is classified as paroxysmal, persistent, and long-standing persistent or permanent. Paroxysmal AF is defined as a continuous AF episode lasting $>30 \mathrm{~s}$ but terminating within 7 days of onset. Persistent AF is defined as a continuous AF episode lasting $>7$ days but $<1$ year. "Long-standing" persistent AF is defined as continuous AF 1 year in duration, in patients in whom rhythm control management is being pursued. Permanent AF is defined as continuous AF for which a therapeutic decision has been made not to pursue sinus rhythm restoration. The mode of termination (spontaneous vs. pharmacological/electrical cardioversion) does not influence the classification. The terms "chronic" AF and "lone" AF are no longer used in both guidelines $[1,2]$.

In addition to this classification, CCS guidelines use another additional classification. They stated that AF may be considered "primary" if the AF represents an established pathophysiological process or "secondary" if caused by a self-limited or acutely reversible precipitant $[2,5]$. Secondary AF can be further dichotomized on the basis of the underlying cardiac substrate and risk for AF recurrence into "reversible" or "provoked" AF [6]. Specifically, "reversible AF" defines AF that occurs solely secondary to an acute illness, with little to no abnormal underlying substrate and therefore limited future risk of AF (hyperthyroidism or alcohol intoxication). In contrast, "provoked AF" represents AF that is unmasked by the acute illness, occurring in patients with significant abnormal underlying substrate, and therefore, ongoing risk for AF recurrence (patients developing AF after mitral valve surgery or in the context of a chronic obstructive pulmonary disease exacerbation). In both guidelines, valvular AF includes the group of patients with moderate/severe mitral stenosis and those with mechanical prosthetic heart valve(s) $[1,2]$.

In both guidelines "Clinical AF" is defined as symptomatic or asymptomatic AF that is documented by sur- 
Table 1. Assessment of symptom severity

\begin{tabular}{|c|c|c|}
\hline $\begin{array}{l}\text { Scoring } \\
\text { system }\end{array}$ & Modified EHRA score & CCS-SAF score \\
\hline Grade 0 & - & Asymptomatic \\
\hline Grade 2 & $\begin{array}{l}2 a-\text { mild symptoms (normal daily activities not affected) } \\
2 b-\text { moderate symptoms (normal daily activities not affected but patient troubled by symptoms) }\end{array}$ & Modest effect on QoL \\
\hline Grade 3 & Severe symptoms affecting normal daily activity & Moderate effect on QoL \\
\hline
\end{tabular}

CCS-SAF, Canadian Cardiovascular Society-Severity of Atrial Fibrillation; EHRA, European Heart Rhythm Association; QoL, quality of life.

face electrocardiogram (ECG) (at least seconds on ambulatory monitor, including wearable-recorded ECG or a 12-lead ECG). Subclinical AF refers to asymptomatic individuals whose pacemaker or implantable cardioverterdefibrillator interrogation reveals "atrial high rate episode," and in whom AF has not been detected on surface ECG. Both guidelines point out the importance of opportunistic screening in AF. ESC guidelines for AF recommend opportunistic screening for AF in patients $\geq 65$ years old, hypertensive patients, and in patients with obstructive sleep apnea. ESC guidelines state that systematic ECG screening should be considered to detect AF in patients aged $\geq 75$ years, or those at high risk of stroke. CCS guidelines for AF recommend that opportunistic screening for AF should be conducted in patients $\geq 65$ years of age at the time of medical encounters (strong recommendation; low-quality evidence). They stated that screening can be efficiently and cost effectively performed using opportunistic pulse checks during routine medical encounters; consideration can also be made to use rhythm-based devices.

\section{Assessment of Symptom Severity and Structured Characterization}

The ESC and CCS guidelines place considerable importance on AF-related symptoms and suggest the use of a symptom score to guide decisions for management of $\mathrm{AF}$, treatments, and patient assessment $[1,2,7,8]$.The ESC guidelines recommend the modified European Heart Rhythm Association (EHRA ) score, which classifies symptoms as none (score 1), mild (score 2A), moderate (score $2 \mathrm{~B}$ ), severe (score 3), and disabling (score 4) [1]. The CCS guidelines recommend the CCS Severity of Atrial Fibrillation (CCS-SAF) scoring system, which grades symptom status as $0-4$ corresponding to asymptomatic, minimal, mild, moderate, and severe effect on QoL [2]. Assessment of symptom severity in these 2 guidelines is summarized in Table 1.

Importantly in recent ESC guidelines for AF, it has been clearly emphasized that all patients diagnosed with AF should undergo a "structured characterization," which includes stroke risk, symptom severity, AF burden, and AF substrate assessment [1,9]. Document proposed 4S-AF scheme (stroke risk, symptom severity, severity of AF burden, and substrate severity) which includes 4 AFrelated domains. Assessment tools/classifications pertinent to specific domains (e.g., stroke-risk scores, symptom scores, clinical factors, and imaging modalities) can be easily fitted in, but the 4S-AF has great potential for future refinements guided by advances in technology, and the most appropriate descriptors of AF domains are yet to be defined. Given the descriptors of AF included in the $4 \mathrm{~S}$-AF scheme, the structured characterization of AF patients using $4 \mathrm{~S}$ - $\mathrm{AF}$ could also provide prognostic information, but the clinical utility and prognostic value of the 4S-AF scheme need extensive validation in different AF cohorts and clinical settings $[1,9]$. In addition, ESC guidelines stated the importance of atrial cardiomyopathy. Important progress in understanding AF mechanisms and thrombogenicity reconsiders the role of atrial cardiomyopathy. AF is not only a risk factor for but also a marker of atrial cardiomyopathy, which could explain the lack of temporal relationship between detected AF and stroke [1]. The diagnostic algorithm for atrial cardiomyopathy should follow a stepwise approach, identifying risk factors for atrial cardiomyopathy, atrial electrical and mechanical dysfunction, and increased thrombotic risk. More data are needed to define prognostic and treatment implications of different types of atrial cardiomyopathy $[10,11]$. 
Table 2. Stroke-risk prediction algorithms

\begin{tabular}{lll}
\hline & $\begin{array}{l}\text { ESC guidelines, } \\
\text { modified } \mathrm{CHA}_{2} \mathrm{DS}_{2} \text {-VASc }\end{array}$ & $\begin{array}{l}\text { CCS guidelines, } \\
\text { "CCS algorithm" (CHADS-65) }\end{array}$ \\
\hline $\begin{array}{ll}\text { Congestive heart failure } \\
\text { Hypertension }\end{array}$ & 1 & 1 \\
Age $\geq 75$ years & 1 & 1 \\
Diabetes mellitus & 2 & Same as age >65 years \\
Stroke, TIA, or systemic embolism & 1 & 1 \\
Vascular disease (peripheral or coronary) & 2 & $\mathrm{n} / \mathrm{a}$ \\
Age $>65$ years for CHADS-65 & 1 & 1 \\
Age 65-74 years for $\mathrm{CHA}_{2} \mathrm{DS}_{2}$-VASc & 1 & $\mathrm{n} / \mathrm{a}$ \\
Female sex & $1^{*}$ & \\
\hline
\end{tabular}

CCS, Canadian Cardiovascular Society; ESC, European Society of Cardiology; n/a, not applicable; $\mathrm{CHA}_{2} \mathrm{DS}_{2}-\mathrm{VASC}_{\text {, }}$ Congestive heart failure, Hypertension, Age $\geq 75$ years, Diabetes mellitus, Stroke, Vascular disease, Age 65-74 years, Sex category (female) score; CHADS-65, Congestive heart failure, Hypertension, Age $>65$ years, Diabetes mellitus, Stroke score. ${ }^{*}$ Not counted in the absence of other risk factors.

Table 3. Antithrombotic recommendations in ESC and CCS guidelines for AF [1, 2]

\begin{tabular}{lll}
\hline $\begin{array}{l}\text { Modified } \mathrm{CHA}_{2} \mathrm{DS}_{2} \text {-VASc or } \\
\text { "CCS algorithm" (CHADS-65) }\end{array}$ & ESC guidelines & CCS guidelines \\
\hline Score 0 & No antithrombotic (I, A) & No antithrombotic* \\
Score 1 & OAC for male (Ila, B) & OAC (strong recommendation) \\
Score $\geq 2$ & OAC for both sexes (I) & OAC (strong recommendation) \\
\hline
\end{tabular}

CCS, Canadian Cardiovascular Society; ESC, European Society of Cardiology; n/a, not applicable; OAC, oral anticoagulant; $\mathrm{CHA}_{2} \mathrm{DS}_{2}-\mathrm{VASC}$, Congestive heart failure, Hypertension, Age $\geq 75$ years, Diabetes mellitus, Stroke, Vascular disease, Age 65-74 years, Sex category female) score; CHADS-65, Congestive heart failure, Hypertension, Age $>65$ years, Diabetes mellitus, Stroke score; ASA, acetylsalicylic acid. * CCS recommends ASA in patients with coronary or vascular disease, but no other risk factors for stroke.

\section{Stroke Prevention, Risk Scores, and Anticoagulation Strategies}

Lack of proper oral anticoagulation (OAC) in $\mathrm{AF}$ is associated with a 5 -fold increased risk of strokes and systemic embolism, which are generally more severe (morbidity and mortality) and more recurrent than non-AF strokes $[12,13]$. Various stroke risk-prediction scores and schemes have been developed to assist clinical decision-making $[1,2]$. Both of the ESC and CCS guidelines have a slightly different approach to stroke risk stratification (Table 2).

Common stroke-risk factors are summarized in the clinical risk factor-based Congestive heart failure, Hypertension, Age $\geq 75$ years, Diabetes mellitus, Stroke, Vascular disease, Age 65-74 years, Sex category $\left(\mathrm{CHA}_{2} \mathrm{DS}_{2^{-}}\right.$ $\mathrm{VASc}[\mathrm{female}]$ ) score. Although the ESC was the 1st to adopt $\mathrm{CHA}_{2} \mathrm{DS}_{2}-\mathrm{VASc}$, the most recent guidelines have modified the consideration of female sex as a stroke-risk modifier rather than a risk factor $[1,3]$. ESC guidelines for AF states that $\mathrm{CHA}_{2} \mathrm{DS}_{2}$-VASc clinical stroke-risk score should be used to identify patients at "low risk" $\left(\mathrm{CHA}_{2} \mathrm{DS}_{2}\right.$-VASc score $=0$ in men, or 1 in women $)$, who should not be offered antithrombotic therapy. Antiplatelet therapy alone is not recommended for stroke prevention in AF (Class III). OAC is recommended for stroke prevention in AF patients with $\mathrm{CHA}_{2} \mathrm{DS}_{2}$-VASc score $\geq 2$ in men or $\geq 3$ in women, and it should be considered in patients with a $\mathrm{CHA}_{2} \mathrm{DS}_{2}$-VASc score of 1 in men or 2 in women, with treatment individualized based on net clinical benefit and patient values/preferences [1]. On the other hand, the CCS guidelines do not consider female sex or vascular disease as indications for OAC. Instead, the CCS retained the $\mathrm{CHADS}_{2}$ framework lowered the 
Table 4. Antithrombotic regimens with concomitant CAD

\begin{tabular}{|c|c|c|c|}
\hline \multirow{3}{*}{ NOAC as the OAC } & \multicolumn{2}{|l|}{ ESC guidelines } & \multirow{3}{*}{$\frac{\text { CCS guidelines }}{\text { Yes }}$} \\
\hline & \multicolumn{2}{|l|}{ Yes } & \\
\hline & Low bleeding risk/high thrombotic risk & High bleeding risk/low thrombotic risk & \\
\hline Stable CAD & OAC & OAC & $\begin{array}{l}\text { CHADS- } 65=0-\text { ASA } \\
\text { CHADS- } 65 \geq 1-\text { OAC }\end{array}$ \\
\hline \multicolumn{4}{|l|}{ Elective PCI } \\
\hline 0-1st week & TT & $\Pi$ & $\mathrm{OAC}+\mathrm{C}$ \\
\hline 1st week-1st month & TT & $\mathrm{OAC}+\mathrm{P} 2 \mathrm{Y} 12$ inhibitor & $\mathrm{OAC}+\mathrm{C}$ \\
\hline 1st-3rd month & $\mathrm{OAC}+\mathrm{P} 2 \mathrm{Y} 12$ inhibitor & $\mathrm{OAC}+\mathrm{P} 2 \mathrm{Y} 12$ inhibitor & $\mathrm{OAC}+\mathrm{C}$ \\
\hline 3rd-6th month & $\mathrm{OAC}+\mathrm{P} 2 \mathrm{Y} 12$ inhibitor & $O A C^{*}$ & $\mathrm{OAC}+\mathrm{C}$ \\
\hline Lifelong & OAC & OAC & OAC \\
\hline \multicolumn{4}{|l|}{ ACS without $\mathrm{PCI}$} \\
\hline 0-6th month & $\mathrm{OAC}+(\mathrm{ASA}$ or preferentially $\mathrm{P} 2 \mathrm{Y} 12$ inhibitor) & $\mathrm{OAC}+(\mathrm{ASA}$ or preferentially $\mathrm{P} 2 \mathrm{Y} 12$ inhibitor) & $\mathrm{OAC}+\mathrm{C}$ \\
\hline 6th-12th month & $\mathrm{OAC}+(\mathrm{ASA}$ or preferentially $\mathrm{P} 2 \mathrm{Y} 12$ inhibitor) & $\mathrm{OAC}^{*}$ & $\mathrm{OAC}+\mathrm{C}$ \\
\hline Lifelong & OAC & OAC & OAC \\
\hline \multicolumn{4}{|l|}{$\mathrm{ACS}$ and $\mathrm{PCl}$} \\
\hline 0-1st week & Tा & $\pi$ & $\pi$ \\
\hline 1st week-1st month & $\mathrm{TT}$ & $\mathrm{OAC}+\mathrm{P} 2 \mathrm{Y} 12$ inhibitor & $\pi$ \\
\hline 1st-6th month & $\mathrm{OAC}+\mathrm{P} 2 \mathrm{Y} 12$ inhibitor & $\mathrm{OAC}+\mathrm{P} 2 \mathrm{Y} 12$ inhibitor & $\mathrm{OAC}+\mathrm{C}$ \\
\hline 6th-12th month & $\mathrm{OAC}+\mathrm{P} 2 \mathrm{Y} 12$ inhibitor & $\mathrm{OAC}+\mathrm{P} 2 \mathrm{Y} 12$ inhibitor & $\mathrm{OAC}+\mathrm{C}$ \\
\hline Lifelong & OAC & OAC & OAC \\
\hline
\end{tabular}

ACS, acute coronary syndrome; ASA, acetylsalicylic acid; C, clopidogrel; CAD, coronary artery disease; CCS, Canadian Cardiovascular Society; CHADS-65, Congestive heart failure, Hypertension, Age $>65$ years, Diabetes mellitus, Stroke score; ESC, European Society of Cardiology; NOAC, non-VKA oral anticoagulant; $\mathrm{OAC}$, oral anticoagulation; $\mathrm{PCl}$, percutaneous coronary intervention; $\mathrm{T}$, triple therapy. ${ }^{*}$ Treatment modifications depending on individual patient's ischemic and bleeding risks.

age criterion to 65 years (“CHADS-65” score) [2]. The rationale underlying these modifications derives largely from the Danish national cohort study [14], in which age 65-74 years was a stronger predictor of stroke than hypertension or diabetes, and at 10 -year follow-up age $>65$ years was the 3 rd strongest predictor of stroke (HR 3.12) behind age $>75$ years (HR 3.38) and prior stroke/TIA (HR 10.44). Conversely, neither female sex nor vascular disease was independently associated with an increased risk of stroke in this series. Because of these variations in risk assessment, the treatment-threshold for use of OAC differs between ESC and CCS guidelines for AF (Table 3).

Another key difference among the guidelines pertains to the antithrombotic agent recommended. The ESC has entirely eliminated acetylsalicylic acid (ASA) from the guidelines, judging that ASA has no role for stroke prevention in AF (Class III recommendation) based on its limited efficacy and significant bleeding risk $[1,3]$. The CCS recommends ASA in patients with coronary or vascular disease, but no other risk factors for stroke [2].

In both ESC and CCS guidelines for AF, patients with $\mathrm{AF}$ and risk factors for stroke need to be treated with OAC for stroke prevention. In nonvitamin $\mathrm{K}$ antagonist oral anticoagulants (NOAC)-eligible patients, NOACs are preferred over vitamin $\mathrm{K}$ antagonists. In both of these recent guidelines, there are new recommendations regarding anticoagulation and cardioversion. ESC guidelines state that in patients with a definite duration of $\mathrm{AF} \leq 24 \mathrm{~h}$ and a very low stroke risk $\left(\mathrm{CHA}_{2} \mathrm{DS}_{2}\right.$-VASc of 0 in men or 1 in women) post-cardioversion anticoagulation for 4 weeks may be omitted [1]. CCS guidelines suggest that pharmacological or electrical cardioversion of symptomatic $\mathrm{AF}$ without at least 3 weeks of previous therapeutic anticoagulation (or TEE) be reserved for patients with NVAF who present with a clear onset of AF within $12 \mathrm{~h}$ in the absence of recent stroke or TIA; or patients with NVAF and a CHADS 2 score of 0 or 1 who present after $12 \mathrm{~h}$ but within $48 \mathrm{~h}$ of AF onset [2].

Importantly, the role of perioperative bridging has been diminished in both ESC and CCS guidelines with the emergence of NOACs. Moreover, several randomized studies including the BRIDGE trial demonstrated that bridging has an unfavorable risk/benefit ration in the majority of cases $[1,2,15]$.

The left-atrial appendage (LAA) is believed to be the major source of AF-related thromboembolism. Exclusion 
of the LAA, either surgically or with percutaneous devices, has been recommended to reduce the risk of stroke and/or peripheral thromboembolism in AF patients. Both ESC and CCS guidelines suggest that LAA occlusion may be considered for stroke prevention in patients with $\mathrm{AF}$ and contraindications for long-term anticoagulant treatment (e.g., intracranial bleeding without a reversible cause). Similarly, both guidelines suggest that surgical LAA occlusion may be considered for stroke prevention in patients with $\mathrm{AF}$ who are at moderate-to-high risk of stroke and have contraindications to $\mathrm{OAC}$, and who are not suitable for percutaneous LAA occlusion.

Both guidelines recommend a risk score-based bleeding risk assessment (HAS-BLED) to help identify patients at high risk of bleeding who should be scheduled for more frequent clinical follow-up. Estimated bleeding risk, in the absence of absolute contraindications to OAC, should not in itself guide treatment decisions to avoid using $\mathrm{OAC}$ $[1,2]$. As a notable difference, ESC guidelines reduced the level of recommendation and evidence of HAS-BLED and highlights the importance of nonmodifiable, modifiable risk factors, and biomarkers, including growth differentiation factor-15, cystatin C, high-sensitivity troponin $\mathrm{T}$ (cTnT-hs), von Willebrand factor, and other coagulation markers [1].

\section{OAC Therapy and CAD}

The problem associated with concomitant $\mathrm{AF}$ and coronary artery disease (CAD) is that OAC is usually indicated for the prevention of AF-related stroke and systemic embolism, whereas antiplatelet therapy is indicated for the prevention of coronary events, and the combination of OAC/antiplatelets can substantially increase bleeding risk. For patients with an elevated risk of stroke, both the ESC and CCS adapt their antithrombotic recommendations according to the risk of coronary events (ranging from stable CAD to elective percutaneous coronary intervention (PCI), to acute coronary syndrome (ACS) without PCI, to ACS with PCI) $[1,2,16,17]$. Both guidelines for $\mathrm{AF}$ recognize the increased bleeding risk associated with the combined use of OAC and antiplatelet therapy, and therefore put forward OAC alone for those with stable $\mathrm{CAD}$, as well as minimizing the intensity and duration of combined prescription whenever feasible $[1,2]$. A substantial difference between the ESC and CCS recommendations hubs on the retention of triple therapy (TT) for all patients at risk of AF-related stroke undergoing stent implantation in the ESC guidelines (with TT-duration depending on bleeding risk), whereas the CCS guidelines recommend TT for only ACS patients undergoing PCI (Table 4).

For patients with ACS but no PCI both societies recommend dual therapy (OAC + antiplatelet) for 12 months. The CCS recommends the combination of OAC + clopidogrel, whereas the ESC concludes that either ASA or preferentially $\mathrm{P} 2 \mathrm{Y} 12$ inhibitor is an appropriate in combination with OAC. Both the CCS and ESC recommend NOACs in preference to vitamin Kantagonists (excluding patients with mechanical heart valves or moderate-to-severe mitral stenosis). Both society guidelines recommend preferably clopidogrel as part of TT-regimens or OAC + antiplatelet regimen $[1,2]$. As a notable difference between these 2 guidelines, patients with ACS and AF undergoing an uncomplicated PCI, early cessation ( $\leq 1$ week) of ASA and continuation of dual therapy with an OAC and a P2Y12 inhibitor (preferably clopidogrel) for up to 12 months is recommended in ESC guidelines for AF. ESC guidelines for AF suggested that TT with ASA, clopidogrel, and an OAC for $>1$ week after an ACS should be considered when risk of stent thrombosis outweighs the bleeding risk, with the total duration $(\leq 1$ month) [1].

\section{Rate Control}

Rate control is important for all patients with AF to improve arrhythmia-related symptoms, exercise tolerance, and QoL, as well as to prevent tachycardiomyopathy $[1,2,18,19]$. Rate control is an integral part of AF management and is often sufficient to improve AF-related symptoms. There are major differences among the ESC and CCS guidelines with respect to heart rate targets. The optimal heart rate target in AF patients is unclear. In the Race Control Efficacy in Permanent Atrial Fibrillation (RACE) II RCT of permanent AF patients, there was no difference in a composite of clinical events, functional class, or hospitalizations between the strict (target heart rate $<80$ beats per minute [bpm] at rest and $<110 \mathrm{bpm}$ during moderate exercise) and lenient (heart rate target $<110 \mathrm{bpm}$ ) arm [20, 21], similar to an analysis from the Atrial Fibrillation Follow-up Investigation of Rhythm Management (AFFIRM ) and RACE trials [22]. Therefore, lenient rate control is an acceptable initial approach, regardless of HF status (with the exception of tachycardia-induced cardiomyopathy), unless symptoms call for stricter rate control. The ESC guidelines are most consistent with RACE II, suggesting treatment initiation if rest-
86

Cardiology 2022;147:81-89 DOI: $10.1159 / 000519465$
Okutucu/Gorenek 
Table 5. AADs for long-term rhythm control

\begin{tabular}{|c|c|c|}
\hline AADs & ESC guidelines & CCS guidelines \\
\hline Amiodarone & $\begin{array}{l}\text { Recommended in all patients, including HFrEF, but other } \\
\text { AADs should be considered 1st whenever possible (class I) }\end{array}$ & $\begin{array}{l}\text { Can be used in all populations, including HF and } \\
\text { CAD. Consider alternative AADs or ablation rather } \\
\text { than long-term amiodarone }\end{array}$ \\
\hline \multirow[t]{2}{*}{ Dronedarone } & \multirow{2}{*}{$\begin{array}{l}\text { Recommended in patients with normal or mildly impaired } \\
\text { left ventricular EF, or HFpEF, ischemic, or VHD (class I) }\end{array}$} & Can be used in CAD or in absence of HF \\
\hline & & $\begin{array}{l}\text { Should be used with caution in combination with } \\
\text { digoxin }\end{array}$ \\
\hline \multirow[t]{2}{*}{$\begin{array}{l}\text { Propafenone } \\
\text { (class } 1 C \text { agents) }\end{array}$} & $\begin{array}{l}\text { Recommended in patients with normal left ventricular } \\
\text { function and without structural heart disease (left } \\
\text { ventricular hypertrophy, ischemia) (class I) }\end{array}$ & Can be used in the absence of HF or CAD \\
\hline & $\begin{array}{l}\text { If flecainide, concomitant atrioventricular nodal blocking } \\
\text { drug (class Ila) }\end{array}$ & $\begin{array}{l}\text { Class } 1 C \text { agents should be combined with } \\
\text { atrioventricular nodal blocking agent. To be used } \\
\text { with caution in patients with left ventricular } \\
\text { hypertrophy }\end{array}$ \\
\hline Sotalol & $\begin{array}{l}\text { May be considered in patients with normal left ventricular } \\
\text { function or ischemic heart disease. QT interval, potassium, } \\
\mathrm{CrCl} \text {, and other proarrhythmia risk factors should be closely } \\
\text { monitored (class Ilb) }\end{array}$ & $\begin{array}{l}\text { Use caution in patients with high-risk features for } \\
\text { torsade de pointes (elderly, female, chronic kidney } \\
\text { disease, diuretics, and electrolyte imbalance) }\end{array}$ \\
\hline
\end{tabular}

AADs, anti-arrhythmic drugs; CAD, coronary artery disease; CCS, Canadian Cardiovascular Society; EF, ejection fraction; ESC, European Society of Cardiology; HF, heart failure; HFpEF, HF with preserved ejection fraction; HFrEF, HF with reduced ejection fraction; VHD, valvular heart disease.

ing HR $>110$ bpm (IIa recommendation). The recent ESC guidelines recommend that the $\mathrm{HR}$ target revert to $<80$ $\mathrm{bpm}$ at rest in the presence of persistent symptoms or tachycardia-induced cardiomyopathy or need for biventricular pacing. The CCS guidelines define the resting HR target as $<100 \mathrm{bpm}$, the rationale being the observation that resting HRs $>100 \mathrm{bpm}$ were associated with adverse outcomes in AFFIRM and RACE, and very few patients in RACE II had a resting HR $>100 \mathrm{bpm}$ (so the safety of resting HRs between 100 and $110 \mathrm{bpm}$ could not be conclusively tested) [2].

\section{Rhythm Control}

Both ESC and CCS guidelines are similar regarding the rhythm control strategy which refers to attempts to restore and maintain sinus rhythm, and may engage a combination of treatment approaches, including cardioversion, anti-arrhythmic medication, and catheter ablation, along with an adequate rate control, anticoagulation therapy, and comprehensive cardiovascular prophylactic therapy (upstream therapy, including lifestyle and sleep apnea management) [1,2,23-25]. Based on the currently available evidence from RCTs, both ESC and CCS guidelines clearly stated that the primary indication for rhythm control is to reduce AF-related symptoms and improve QoL $[1,2]$. Both guidelines highlight the importance of the decision to initiate long-term anti-arrhythmic drug (AAD) which needs to balance symptom burden, possible adverse drug reactions, particularly drug-induced proarrhythmia or extracardiac side-effects, and patient preferences. Recommendations of both guidelines regarding the use of AADs for long-term rhythm control are shown in Table 5.

Catheter ablation of AF has emerged as an important therapeutic modality for this common arrhythmia [26]. The aim of catheter ablation was to eliminate the triggers and substrate responsible for the initiation and maintenance of AF $[1,26]$. The recommendations of the ESC and CCS guidelines for AF are in general agreement, with each providing a strong recommendation for $\mathrm{AF}$ catheter ablation with pulmonary vein isolation for rhythm control after 1 failed or intolerant class I or III AADs, to im- 
prove symptoms of AF recurrences in patients with paroxysmal AF, or persistent AF. In addition, AF catheter ablation is recommended to reverse LV dysfunction in AF patients when tachycardia-induced cardiomyopathy is highly probable, independent of their symptom status. Both ESC and CCS guidelines for AF emphasize the importance of high annual procedure-volumes for operators and sites to preserve optimal technical competence for AF ablation $[1,2]$.

There is significant variation in the surgical management of AF, both in terms of procedural approach and clinical indications $[1,2]$. The evidence supporting surgical AF ablation with the standard MAZE procedure is best established for concomitant procedures during mitral valve intervention, as endocardial access to the left atrium is essential for the procedure. Both guidelines recommend adjuvant surgical ablation for AF-patients undergoing cardiac surgery $[1,2]$. Stand-alone thoracoscopic, including hybrid surgical ablation, procedures should be considered in patients who have symptomatic paroxysmal or persistent AF refractory to AAD and have failed percutaneous AF ablation, or with evident risk factors for catheter ablation failure, to maintain long-term sinus rhythm. The decision must be supported by an experienced team of electrophysiologists and surgeons.

\section{Comprehensive Care and Risk Factor Modification}

Both ESC and CCS guidelines for AF highlight the importance of comprehensive care and risk factor modification. According to ESC guidelines for AF, the principles of management of AF can be aligned with the components of integrated or holistic management of AF as follows: "A" avoid stroke with anticoagulation, "B" better symptom management with patient-centered decisions on rate or rhythm control, and " $\mathrm{C}$ " cardiovascular risk and comorbidity management, including lifestyle changes. This is referred to as the Atrial fibrillation Better Care (ABC) pathway [27-29]. In current ESC guidelines, this holistic approach is integrated to the CC-ABC strategy. In this strategy, 1 st $C$ letter stands for confirming AF with a 12-lead ECG or a rhythm strip showing AF pattern for $\geq 30$ s. 2nd C letter signifies characterized AF with the 4 S$\mathrm{AF}$ scheme and $\mathrm{T}$ letter means treat AF with the simple $\mathrm{ABC}$ holistic pathway ("A" Anticoagulation/Avoid stroke; "B" Better symptom management; "C" Cardiovascular and Comorbidity optimization). This strategy simplifies integrated care of AF patients across all healthcare levels and among different specialties. Compared with usual care, implementation of the $\mathrm{ABC}$ pathway has been significantly associated with lower risk of all cause death, composite outcome of stroke/major bleeding/cardiovascular death, and 1st hospitalization, lower rates of cardiovascular events, and lower health-related costs [27-29]. Both guidelines recognize that risk factor modification is as an important part of the management of patients with AF. CCS and ESC guidelines clearly give a strong or class I recommendation, respectively, for the identification and management of modifiable cardiovascular risk factors.

Finally, in both ESC and CCS guidelines for AF, there has been increasing recognition of the role of comprehensive risk factor modification in improving outcomes for AF patients. Beyond the comprehensive health benefits, targeted risk factor modification for elements like obesity, alcohol consumption, cigarette smoking, sleep apnea, and physical inactivity have been associated with suppression of AF triggers and amelioration of the arrhythmogenic substrate $[1,2]$.

\section{Conclusion}

The data on diagnosis and management of AF progress rapidly as the evidence base expands and new trials are published. Therefore, there is ongoing demand for updates and new guidelines. The majority of recommendations within recently published ESC and CCS guidelines for AF are similar; however, some differences are observed, particularly when the quality of evidence is moderate or low. Some of these differences reflect the health systems and inherent practice patterns across countries for which these society guidelines were written. Other areas reflect the differential interpretation in clinical evidence, studies, and trials that have shaped contemporary AF management. More research and randomized controlled studies on major gaps identified in current guidelines will further clarify our management strategies in AF. Better detection of AF, stroke and bleeding prediction, and emergence of newer antithrombotics and AADs with better safety and efficacy profiles will probably further modify our clinical practice in the field of AF. Consideration of these differences highlights areas of inadequate and conflicting evidence that call out for further research to strengthen the evidence base.

\section{Conflict of Interest Statement}

The authors have no conflicts of interest to declare.
Okutucu/Gorenek 


\section{Funding Sources}

This research received no specific grant from any funding agency in the public, commercial, or not-for-profit sectors.

\section{Author Contributions}

Both authors equally contributed to the conception, design, interpretation, literature review, and writing of the manuscript.

\section{References}

1 Hindricks G, Potpara T, Dagres N, Arbelo E, Bax JJ, Blomstrom-Lundqvist C, et al. 2020 ESC Guidelines for the diagnosis and management of atrial fibrillation developed in collaboration with the European Association for Cardio-Thoracic Surgery (EACTS). Eur Heart J. 2021;42(5):373-498.

2 Andrade JG, Aguilar M, Atzema C, Bell A, Cairns JA, Cheung CC, et al. The 2020 Canadian Cardiovascular Society/Canadian Heart Rhythm Society Comprehensive Guidelines for the management of atrial fibrillation. Can J Cardiol. 2020;36(12):1847-948.

3 Kirchhof P, Benussi S, Kotecha D, Ahlsson A, Atar D, Casadei B, et al. 2016 ESC Guidelines for the management of atrial fibrillation developed in collaboration with EACTS2016. Eur Heart J. 2016;37(38):2893-962.

4 Gillis AM, Skanes AC, CCS Atrial Fibrillation Guidelines Committee. Canadian Cardiovascular Society atrial fibrillation guidelines 2010: implementing GRADE and achieving consensus. Can J Cardiol. 2011;27(1):27-30.

5 Wyse DG, Van Gelder IC, Ellinor PT, Go AS, Kalman JM, Narayan SM, et al. Lone atrial fibrillation: does it exist? J Am Coll Cardiol. 2014;63(17):1715-23.

6 Cheung CC, Andrade JG. Reversible or provoked atrial fibrillation?: the devil in the details. JACC Clin Electrophysiol. 2018;4(4): 563-4.

7 Wynn GJ, Todd DM, Webber M, Bonnett L, McShane J, Kirchhof P, et al. The European Heart Rhythm Association symptom classification for atrial fibrillation: validation and improvement through a simple modification. Europace. 2014;16(7):965-72.

8 Schnabel RB, Pecen L, Rzayeva N, Lucerna M, Purmah Y, Ojeda FM, et al. Symptom burden of atrial fibrillation and its relation to interventions and outcome in Europe. J Am Heart Assoc. 2018;7(11):e007559.

9 Potpara TS, Lip GYH, Blomstrom-Lundqvist C, Boriani G, Van Gelder IC, Heidbuchel H, et al. The 4S-AF Scheme (stroke risk; symptoms; severity of burden; substrate): a novel approach to in-depth characterization (rather than classification) of atrial fibrillation. Thromb Haemost. 2021;121(3):270-8.

10 Goette A, Kalman JM, Aguinaga L, Akar J, Cabrera JA, Chen SA, et al.EHRA/HRS/APHRS/ SOLAECE expert consensus on atrial cardiomyopathies: definition, characterization, and clinical implication. Europace. 2016;18(10): 1455-90.

11 Hirsh BJ, Copeland-Halperin RS, Halperin JL. Fibrotic atrial cardiomyopathy, atrial fibrillation, and thromboembolism: mechanis- tic links and clinical inferences. J Am Coll Cardiol. 2015;65(20):2239-51.

12 Lip GY, Nieuwlaat R, Pisters R, Lane DA, Crijns HJ. Refining clinical risk stratification for predicting stroke and thromboembolism in atrial fibrillation using a novel risk factorbased approach: the euro heart survey on atrial fibrillation. Chest. 2010;137(2):263-72.

13 Pisters R, Lane DA, Marin F, Camm AJ, Lip GY. Stroke and thromboembolism in atrial fibrillation. Circ J. 2012;76(10):2289-304.

14 Olesen JB, Lip GY, Hansen ML, Hansen PR, Tolstrup JS, Lindhardsen J, et al. Validation of risk stratification schemes for predicting stroke and thromboembolism in patients with atrial fibrillation: nationwide cohort study. BMJ. 2011;342:d124.

15 Douketis JD, Spyropoulos AC, Kaatz S, Becker RC, Caprini JA, Dunn AS, et al. Perioperative bridging anticoagulation in patients with atrial fibrillation. N Engl J Med. 2015;373(9):823-33.

16 Ibanez B, James S, Agewall S, Antunes MJ, Bucciarelli-Ducci C, Bueno H, et al. 2017 ESC guidelines for the management of acute myocardial infarction in patients presenting with ST-segment elevation: the task force for the management of acute myocardial infarction in patients presenting with ST-segment elevation of the European Society of Cardiology (ESC). Eur Heart J. 2018;39(2):119-77.

17 Collet JP, Thiele H, Barbato E, Barthelemy O, Bauersachs J, Bhatt DL, et al. 2020 ESC guidelines for the management of acute coronary syndromes in patients presenting without persistent ST-segment elevation. Eur Heart J. 2021;42(14):1289-367.

18 Ulus T, Okyay K, Kabul HK, Özcan EE, Özeke Ö, Altay H, et al. Turkish Society of Cardiology consensus paper on management of arrhythmia-induced cardiomyopathy. Anatol J Cardiol. 2019;21(2):98-106.

19 Al-Khatib SM, Allen LaPointe NM, Chatterjee R, Crowley MJ, Dupre ME, Kong DF, et al. Rate- and rhythm-control therapies in patients with atrial fibrillation: a systematic review. Ann Intern Med. 2014;160(11):760-73.

20 Groenveld HF, Crijns HJ, Van den Berg MP, Van Sonderen E, Alings AM, Tijssen JG, et al. The effect of rate control on quality of life in patients with permanent atrial fibrillation: data from the RACE II (rate control efficacy in permanent atrial fibrillation II) study. J Am Coll Cardiol. 2011;58(17):1795-803.

21 Van Gelder IC, Groenveld HF, Crijns HJ, Tuininga YS, Tijssen JG, Alings AM, et al. Lenient versus strict rate control in patients with atrial fibrillation. N Engl J Med. 2010;362(15): 1363-73.
22 Van Gelder IC, Wyse DG, Chandler ML, Cooper HA, Olshansky B, Hagens VE, et al. Does intensity of rate-control influence outcome in atrial fibrillation? An analysis of pooled data from the RACE and AFFIRM studies. Europace. 2006;8(11):935-42.

23 Gorenek B, Pelliccia A, Benjamin EJ, Boriani G, Crijns HJ, Fogel RI, et al. European Heart Rhythm Association (EHRA)/European Association of Cardiovascular Prevention and Rehabilitation (EACPR) position paper on how to prevent atrial fibrillation endorsed by the Heart Rhythm Society (HRS) and Asia Pacific Heart Rhythm Society (APHRS). Eur J Prev Cardiol. 2017;24(2):4-40.

24 Camm AJ, Kirchhof P, Lip GY, Schotten U, Savelieva I, Ernst S, et al. Guidelines for the management of atrial fibrillation: the task force for the management of atrial fibrillation of the European Society of Cardiology (ESC). Europace. 2010;12(19):1360-420.

25 Camm AJ, Lip GY, De Caterina R, Savelieva I, Atar D, Hohnloser SH, et al. 2012 focused update of the ESC Guidelines for the management of atrial fibrillation: an update of the 2010 ESC Guidelines for the management of atrial fibrillation. Developed with the special contribution of the European Heart Rhythm Association. Eur Heart J. 2012;33(21):271947.

26 Calkins H, Hindricks G, Cappato R, Kim YH, Saad EB, Aguinaga L, et al. 2017 HRS/EHRA/ ECAS/APHRS/SOLAECE expert consensus statement on catheter and surgical ablation of atrial fibrillation: executive summary. Europace. 2018;20(1):157-208.

27 Proietti M, Romiti GF, Olshansky B, Lane DA, Lip GYH. Improved outcomes by integrated care of anticoagulated patients with atrial fibrillation using the simple ABC (atrial fibrillation better care) pathway. Am J Med. 2018;131(11):1359-e6.

28 Yoon M, Yang PS, Jang E, Yu HT, Kim TH, Uhm JS, et al. Improved population-based clinical outcomes of patients with atrial fibrillation by compliance with the simple $\mathrm{ABC}$ (atrial fibrillation better care) pathway for integrated care management: a Nationwide Cohort Study. Thromb Haemost. 2019;119(10): 1695-703.

29 Gorenek B, Halvorsen S, Kudaiberdieva G, Bueno H, Van Gelder IC, Lettino M, et al. Atrial fibrillation in acute heart failure: a position statement from the Acute Cardiovascular Care Association and European Heart Rhythm Association of the European Society of Cardiology. Eur Heart J Acute Cardiovasc Care. 2020;9(4):348-57. 